\title{
Spatial Filtering with a Pyramid Wavefront Sensor on the High Order Testbench
}

\author{
Tyler Banas ${ }^{\mathrm{a}}$, Markus Kasper ${ }^{\mathrm{a}}$, Alfio Puglisi ${ }^{\mathrm{b}}$, and Christophe Vérinaud ${ }^{\mathrm{a}}$ \\ aEuropean Southern Observatory, Karl-Schwarschilder-Straße 2, 85748 Garching bei München, \\ Germany \\ ${ }^{\mathrm{b}}$ Arcetri Astrophysical Observatory, Largo Enrico Fermi 5, 50125 Firenze, Italy
}

\begin{abstract}
Wavefront sensors used in adaptive optics $(\mathrm{AO})$ can misinterpret high spatial frequency wavefront aberrations as low spatial frequency aberrations, known as aliasing. The AO system then corrects for fictitious low-order aberrations, thus adding phase error. Aliasing is a significant contributor to the error budget for XAO. Spatial filtering in the focal plane is a widely accepted practice for Shack-Hartmann Wavefront sensors, ${ }^{1}$ and is currently used on SPHERE and GPI. While simulations have shown that the pyramid wavefront sensor (PWS) design is less suscebtible to aliasing than the Shack-Hartmann design, ${ }^{2,3}$ no thorough experimental tests have previously been conducted to verify these simulations. This article describes experiments undertaken using the High Order Testbench (HOT) at the European Southern Observatory headquarters in Munich to explore the effect of a spatial filter on a PWS. The goal of this investigation is to experimentally demonstrate the degree to which a PWS is susceptible to the adverse effects of aliasing. If spatial filtering improves the PWS performance in terms of some relevant metric (e.g. Strehl ratio, contrast, sensitivity), then the optimal aperture size will be identified. In the end, it is shown that the PWS is quite robust against aliasing, but there remain secondary effects caused by spatial filtering that may have useful applications.
\end{abstract}

Keywords: adaptive optics, pyramid wavefront sensor, aliasing, spatial filtering, coronagraphy, XAO, astronomical instrumentation, wavefront sensing

\section{INTRODUCTION}

\subsection{The problem of aliasing}

An AO system is characterized by the highest spatial frequency $f_{c}$ that it is able to correct. Atmospheric turbulence, however, is not limited to $f_{c}$. All spatial frequencies higher than $f_{c}$ will not be corrected, and thus contribute to the residual wavefront error. However, there is a secondary effect that causes an adaptive optics system to work against itself: aliasing. Aliasing occurs when high order spatial frequencies of the turbulence are detected by the wavefront sensing camera, but are misunderstood as low order spatial frequencies. ${ }^{2}$ The AO system then reconstructs a false phase map, and in the end adds distortion to the wavefront. Figure 1 shows how aliasing causes a DM to smear light in the area that should be well corrected.

Several decades ago, when AO was a nascent technology, aliasing was not of high concern because other factors such as photon noise and the chromatic index of the atmosphere took up a larger portion of the error budget. ${ }^{4}$ As AO systems achieve diffraction-limited imaging, however, aliasing becomes an increasingly dominant factor. Removing the presence of high order spatial frequencies will improve the performance of an AO system.

\subsection{Spatial filtering}

Higher spatial frequency content focuses further away from the PSF core than low spatial frequency content in the focal plane, as shown in Figure 2 using a HeNe laser on HOT. Placing an aperture in the focal plane can remove high spatial frequency aberrations beyond the cutoff frequency of an $\mathrm{AO}$ system. Figure 3 demonstrates this technique.

\footnotetext{
For further information, send correspondence to Tyler Banas: tbanas@eso.org, +49 8932006718
} 


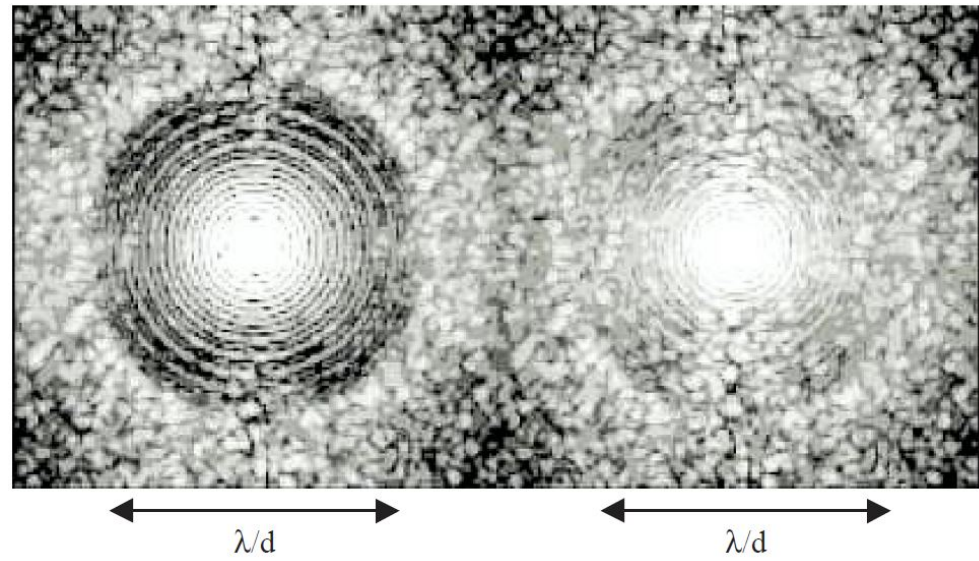

Figure 1: An example of how aliasing can significantly reduce the quality of Airy rings in a PSF and therefore Strehl ratio. ${ }^{2}$

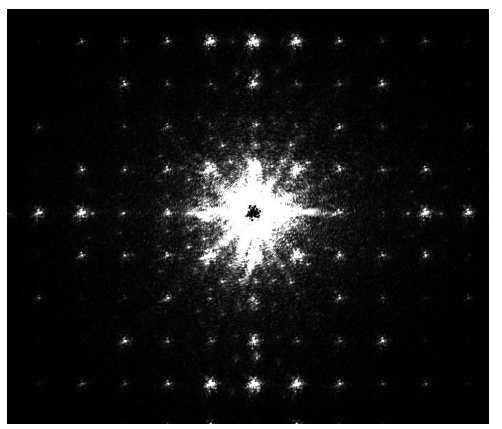

Figure 2: High spatial frequency content far from focal plane core. An aperture mask will block out the photons far away from the PSF core.

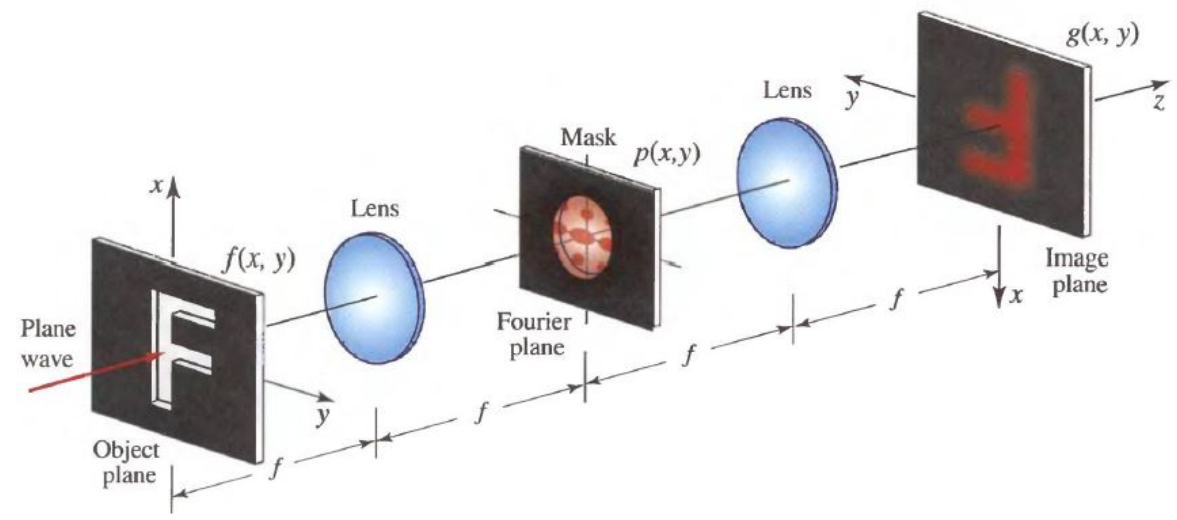

Figure 3: Ray diagram showing how a mask in the focal plane removes high spatial frequency content. ${ }^{5}$

\subsection{The effect of spatial filtering on wavefront sensors}

Many previous studies have shown how implementation of a spatial filter in a focal plane of the wavefront sensing path can be used to significantly reduce the performance-limiting effects of aliasing on the wavefront sensor, thus causing the spatially filtered Shack-Hartmann wavefront sensor to become the most popular type of wavefront sensor at the moment. ${ }^{1}$ Simulations show that spatial filtering could slightly improve the performance of a PWS, but to a lesser extent as with the Shack-Hartmann wavefront sensor.$^{2}$

\section{THE HIGH ORDER TESTBENCH}

HOT was first established in 2006 at the European Southern Observatory headquarters to implement extreme adaptive optic experiments. It was the first testbench to incorporate all the components of a real XAO system while simulating real telescope turbulence conditions. The main components of HOT will here be briefly described, and a photo representation of the testbench is provided in Figure 4.

Turbulence Generator Turbulence is generated using two reflective phase screens with a diameter of $5.0 \mathrm{~cm}$ each. The phase screens are specially manufactured to have reduced low-order aberrations. They are installed on rotating mounts that turn in different directions and at adjustable speeds. This ensures a continuous shift between the two phase screens, providing reliable phase decorrelation. They are positioned in sequence with spherical mirrors of different curvature radii, producing an $\mathrm{f} / 16.8$ beam at the turbulence generator output. 


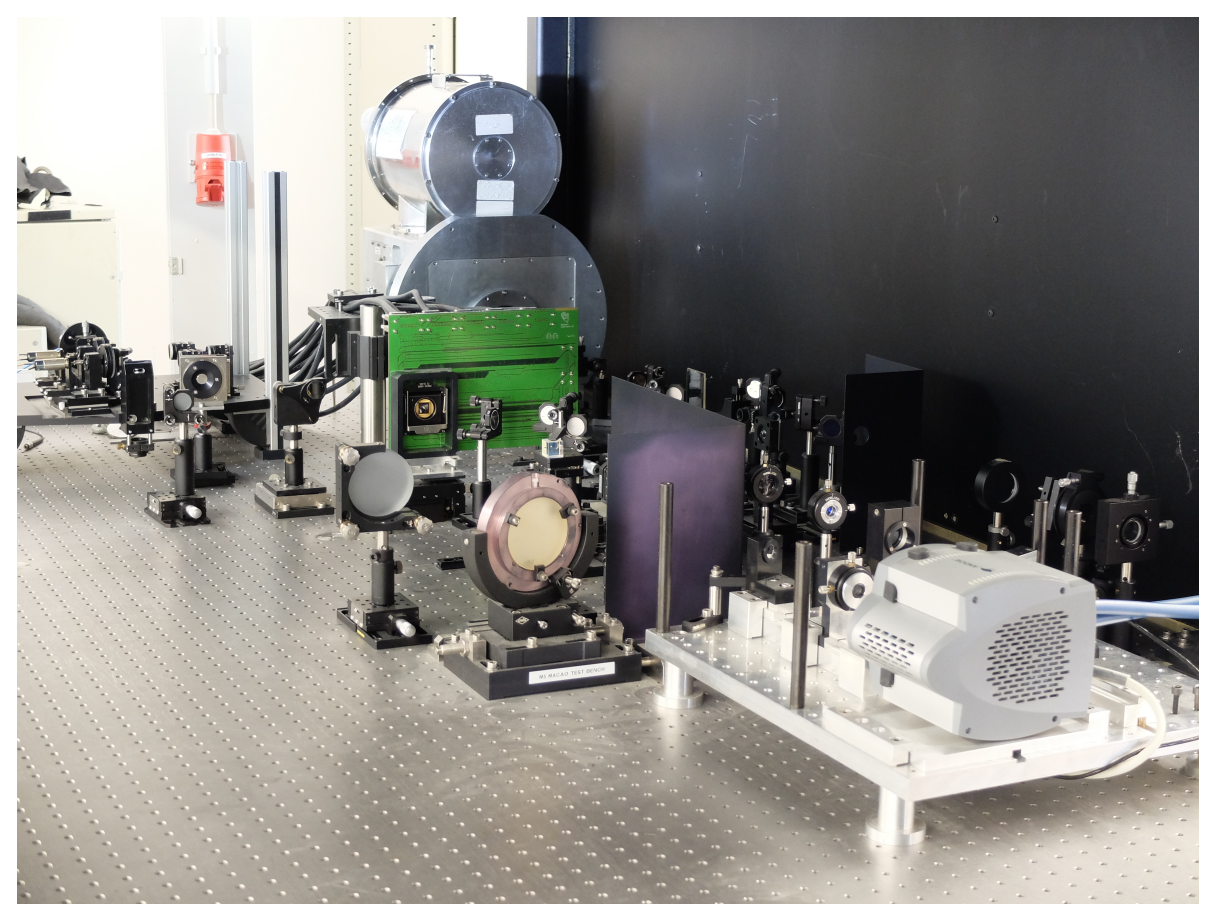

Figure 4: HOT provides a malleable laboratory environment to test novel XAO subsystems and techniques. Primary subsystems are a turbulence generator, two deformable mirrors, a PWS, and a cryogenic infrared camera.

An alternative input with equivalent beam properties is available for bypassing the turbulence generator during alignment.

ALPAO DM52 Deformable Mirror Casually known as the "woofer", this 52-actuator mirror is used to dynamically correct tip-tilt and statically correct low order aberrations of the raw optics.

Boston Micromachines Deformable Mirror Casually known as the "tweeter", this 1024-actuator mirror corrects high order wavefront aberrations. It has a significantly smaller stroke than the ALPAO DM52 (a few $\mu \mathrm{m}$ compared to $100 \mu \mathrm{m})$.

Infrared Test Camera The ITC is a Hawaii array $(1 \mathrm{~K} \times 1 \mathrm{~K})$ with four contiguous quadrants $(512 \times 512)$. The borders of the quadrants are visible in camera images, so the center of the PSF is usually placed inside one of the quadrants. The camera's internal optics are designed to enable a pixel-scale of 5.3 mas/pix. Pixel counts higher than 9000 ADU are considered over-exposed. The read-out noise is below 30 electrons. The ITC has a filter wheel to allow for five different band settings: J-band, H-band, K-band, blind, and no filter.

Pyramid Wavefront Sensor The PWS for the HOT bench is based on the PWS developed for Large Binocular Telescope AO system. The Arcetri Observatory is responsible for the design, integration and first laboratory test of the PWS. The PWS is an independent module installed on a separate board that integrates on the HOT bench. While HOT previously had an additional Shack-Hartmann wavefront sensor, the PWS is now the primary wavefront sensor used on HOT. 


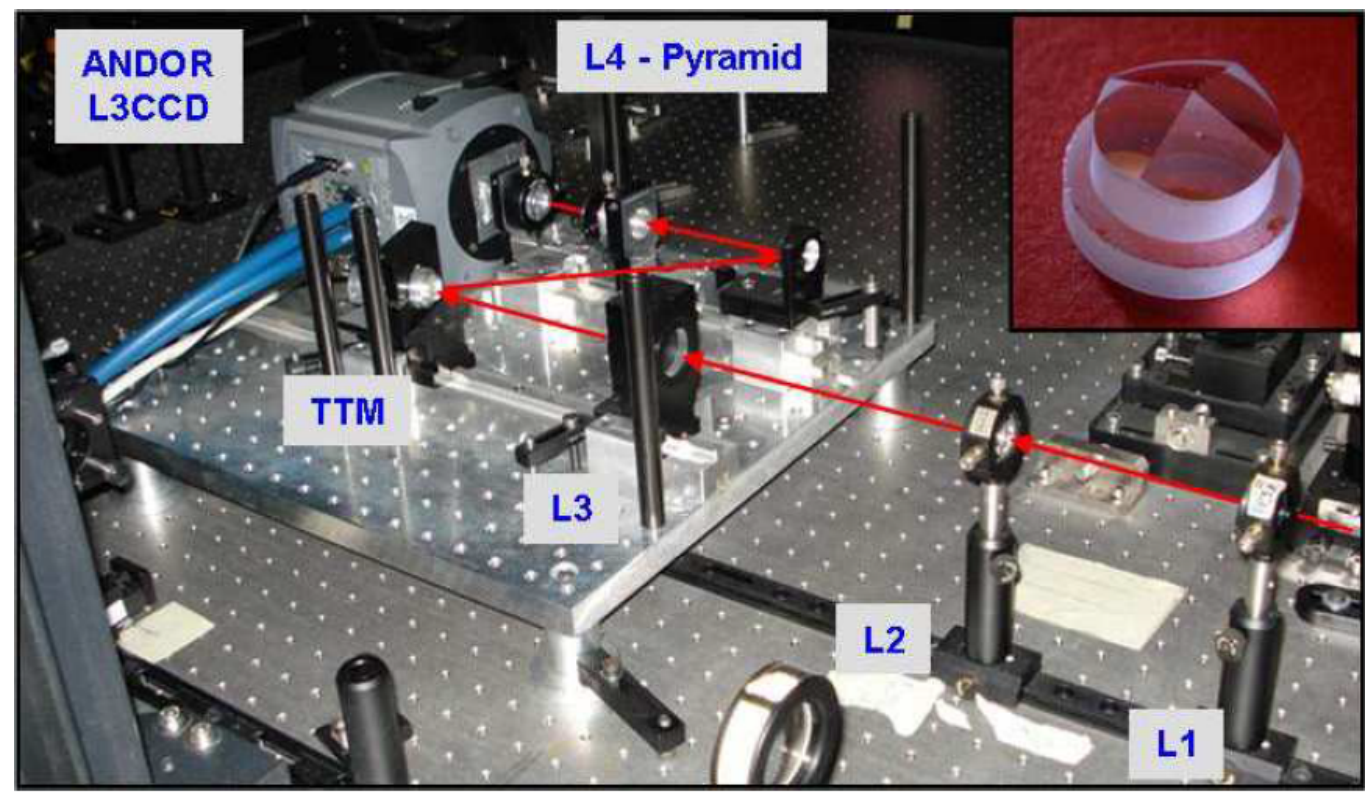

Figure 5: The PWS situated on HOT. The optical board measures $32.5 \times 30.0 \mathrm{~cm}$ and holds the fast tip-tilt mirror, the double pyramid (one pyramid displayed in the upper right), and the Andor camera. ${ }^{6}$

\section{METHODS}

This section outlines the steps that were carried out in order to implement the spatial filtering experiment. The main opto-mechanical step was accurate placement of the aperture mask. Additionally, special PWS and science camera settings were implemented as well.

\subsection{Determination of aperture size}

The primary factor that limits an adaptive optics system's ability to correct high spatial frequencies is either inter-actuator spacing on the deformable mirror or the resolution of the wavefront sensor, depending on which is larger of the two. An ideal spatial filter would block all of the frequency content that an adaptive optics system is not able to correct and pass all of the frequency content that the system is able to correct. To know the size of the ideal spatial filter, the cutoff frequency $f_{o}$ needs to be computed as

$$
f_{o}=f / \# \cdot N_{\text {subap }} \cdot \lambda_{\text {sensing }} \quad,
$$

where $N_{\text {subap }}$ is (in the context of a PWS) the number of pixels across the diameter of one pupil on the wavefront sensing detector. In the case of HOT, $f_{o} \approx 1 \mathrm{~mm}$. Therefore, the minimum size of the spatial filter used for this experiment needs to be at most $1 \mathrm{~mm}$.

\subsection{Procurement of adjustable aperture mask}

Two spatial filters were acquired for this study. The first was a Thorlabs zero-aperture diaphragm. The two leaf closing mechanism is however not practical for two reasons. First, the spacing between the leaves is quite large relative to the beam speed, thus making good placement in the focal plane hard to achieve. Second, the aperture looses its round shape when small.

The second spatial filter is better suited for our purpose. Although not zero minimum, it goes down to 1 $\mathrm{mm}(\lambda / d)$, which is the lowest we need to achieve. Moreover, the aperture has a graduated scale to allow for repeatability of experiments. 


\subsection{Placement of the aperture mask}

Precise placement of the aperture is very important. First of all, vignetting is likely to occur if the aperture is not properly placed. Second, different spatial frequencies are not spatially separated anywhere outside of the focal plane. Thus, this spatial filtering experiment can only be completed if the aperture is located precisely in the focal plane.

Several steps were undertaken to ensure proper placement of the aperture. First, the alignment laser on its brightest setting was used to identify the focal plane with the unaided eye. Note that the laser and the white light have the same focal plane because no lenses (i.e. chromatic optical elements) are used in the common path. Next, a small optical camera was placed in the subsequent focal plane downstream from the aperture mask focal plane. Diffuse white light was positioned to illuminate the aperture mask and produce a picture on the mini-cam. The position of the aperture mask was finely adjusted to produce sharp edges.

It is also necessary to ensure that the focal point of the light beam is located in the center of the aperture mask. For this, a similar technique was used as the one used to ensure that the aperture mask is in the focal plane. The only difference is that, in this case, the laser light was also turned on. Thus, two light sources were in place: the diffuse light shows the outer ring of the aperture mask, which itself needs to be adjusted in the $\mathrm{x}-\mathrm{y}$ plane using the fine tuning mounts so that the focal point of the laser beam is in the center of the mask.

\subsection{Turbulence generator wind speed}

Because HOT currently operates at only $80 \mathrm{~Hz}$ in closed-loop, it is necessary to ensure that the system is not subject to unreasonably fast windspeeds. A modern XAO instrument like SPHERE operates at $>1000 \mathrm{~Hz}$ where high wind speeds are $20 \mathrm{~m} / \mathrm{s}$. Correspondingly, we set the rotational speed of the TG phase screens to a proportional $2 \mathrm{~m} / \mathrm{s}$.

The size of the beam on the phase screens as well as its radial location was determined using paper with $\mathrm{mm}$ grading. The phase screens themselves are $56 \mathrm{~mm}$ in diameter. The average size of the beam on the phase screen is $15 \mathrm{~mm}$ and has an average distance from the center of $17 \mathrm{~mm}$. With $20 \mathrm{~m} / \mathrm{s}$ wind at the $8 \mathrm{~m}$ VLT, the turbulence pattern transverses through 2.5 times the pupil diameter every second; therefore, the phase screen at $17 \mathrm{~mm}$ radius needs to rotate through 2.5 times the average diameter of the beam on the phase screen, or 38 $\mathrm{mm}$. The circumference of the phase screen at $17 \mathrm{~mm}$ radius is $106 \mathrm{~mm}$. This would correspond to 0.36 rotations per second for a AO system working at $1000 \mathrm{~Hz}$, but since HOT currently runs at $<100 \mathrm{~Hz}, 0.036$ rotations per second is appropriate. This corresponds to 2.16 rotations per minute in frequency space.

\subsection{Collect pupils and interaction matrices}

Without dynamic turbulence, one set of reference pupils for the PWS was acquired and optimized. Unique interaction matrices for 300 KarhunenLove modes were collected for spatial filter sizes ranging from $1-10 \lambda / d$ following a $10-50-100-200-300$ mode bootstrapping method. The PWS modulation was set to $6 \lambda / d$.

\subsection{Infrared PSFs with turbulence}

One 0.5 seeing phase screen (with reduced low-order aberrations) on the turbulence generator was set with a wind speed equivalent to $20 \mathrm{~m} / \mathrm{s}$ at the VLT. For varying spatial filter sizes, a sequence of 50 short (too increase the signal to noise ratio) DIT H-band images was collected in closed-loop AO. The loop gain in each case was pushed to 1. All other parameters (e.g., camera settings) were constant.

\subsection{Deep PSF collection}

Collecting a deep PSF is a rather involved task. First of all, it is important that the detector used in such measurements does not saturate. Second, it is important that as many Airy rings as possible are obtained. These are two contradicting goals in terms of instrumental set-up. The solution is to take multiple exposures (at least one in which the central core is not saturated, and at least one in which the third or fourth Airy Rings are clearly visible) and then combine them through post-processing techniques.

Several attempts were made to create a multiple exposure deep PSF, but no appropriate scheme for weighting the different images was found. Further efforts will be dedicated to this task. For now, a non-rigorous stacking of two images for inspection of the PSF structure suffices. 

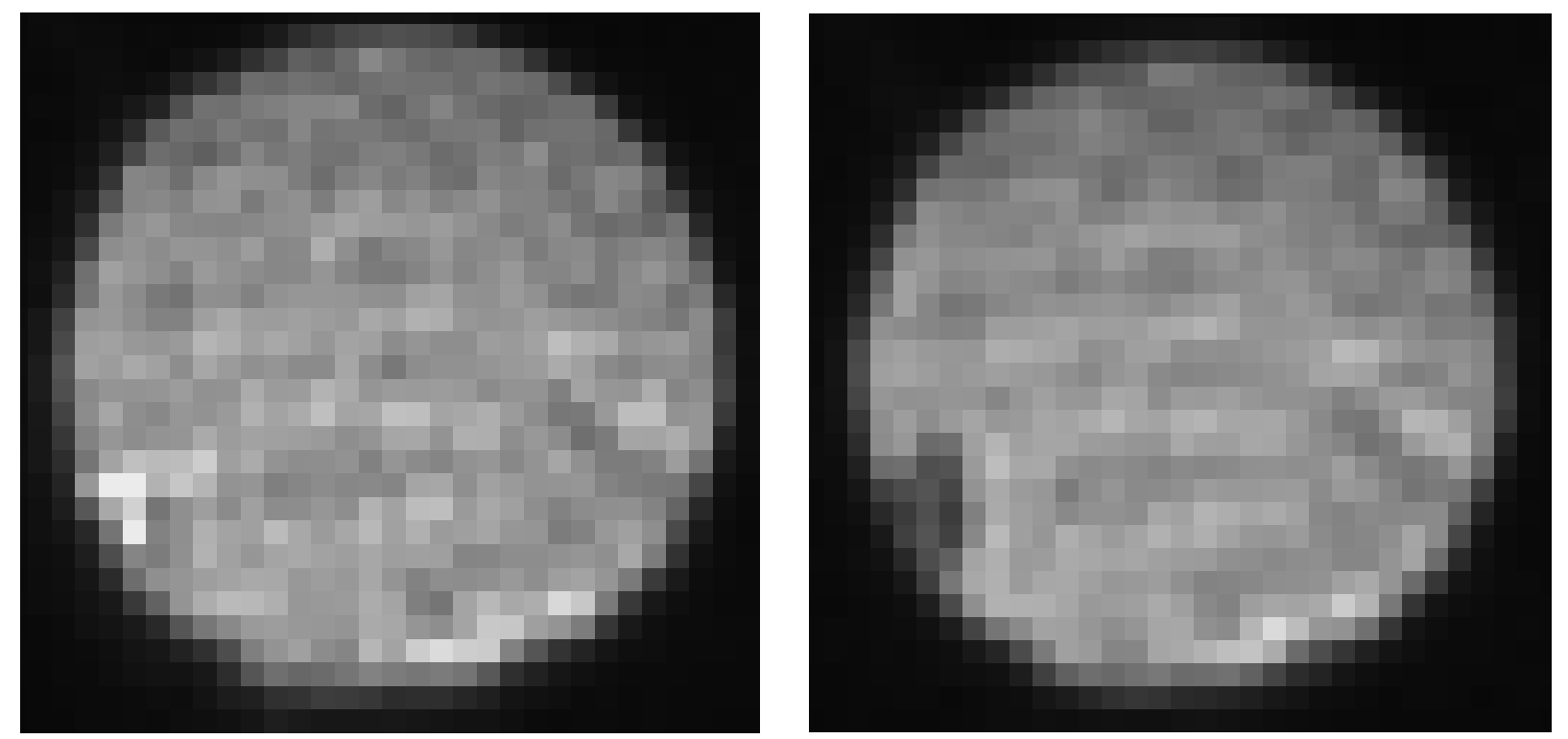

Figure 6: Images of one of the four PWS pupils (left: no spatial filter, right: $1 \lambda / d$ spatial filter), showing how spatial filter blocks light scattered from stuck actuator, as well as other high spatial frequency content.

\section{RESULTS}

\subsection{Immediate drop in RMS wavefront error}

The first observation while having the aperture mask in place is that the RMS wavefront error calculated by the PSF software immediately drops - in one test, from $53 \mathrm{~nm}$ with no spatial filter to $46 \mathrm{~nm}$ with the spatial filter. This suggests that the aperture mask is indeed blocking high spatial frequency content that adds to the RMS wavefront error.

\subsection{Stuck actuator mask}

The aperture mask blocks light scattered by the stuck actuator. Light scattered by a point-like structure in the pupil plane is a plane-like structure in the focal plane. The spatial filter then removes nearly all of the light scattered by the stuck actuators. Poyneer et al., 2014 observed this effect as well on the spatially filtered Shack-Hartmann sensor. ${ }^{7}$ Further research should be undertaken to investigate the effect of using a spatial filter as a mask for stuck actuators.

\subsection{Strehl ratio}

The algorithm used to determine the Strehl ratio of the PSFs obtained in closed loop with and without the field stop for spatial filtering has been implemented in MATLAB. The algorithm calculates an ideal PSF that would have the same total luminosity as the input image. It then finds the maximum intensity of the input image, and divides by the maximum intensity of the ideal PSF. This is the definition of the Strehl ratio.

The presence of a spatial filter has minimal effect on Strehl ratio and PSF structure. Spatial filter sizes between 1.5-10 $\lambda / d$ produced no significant differences in PSF structure or Strehl ratio, suggesting that the PWS is not highly susceptible to aliasing. Figure 7 shows these results in more depth.

\subsection{Contrast levels}

An immediate analysis in terms of contrast for the preceding images does not provide any useful information because the contrast curves are dominated by the Airy rings. The effects of the Airy rings can be mitigated by a coronagraph. To this end, a relatively inexpensive vortex coronagraph was acquired from Thorlabs and installed on HOT. The vortex coronagraph is an $\mathrm{m}=2$ coronagraph optimized for use with $1550 \mathrm{~nm}$ light. 

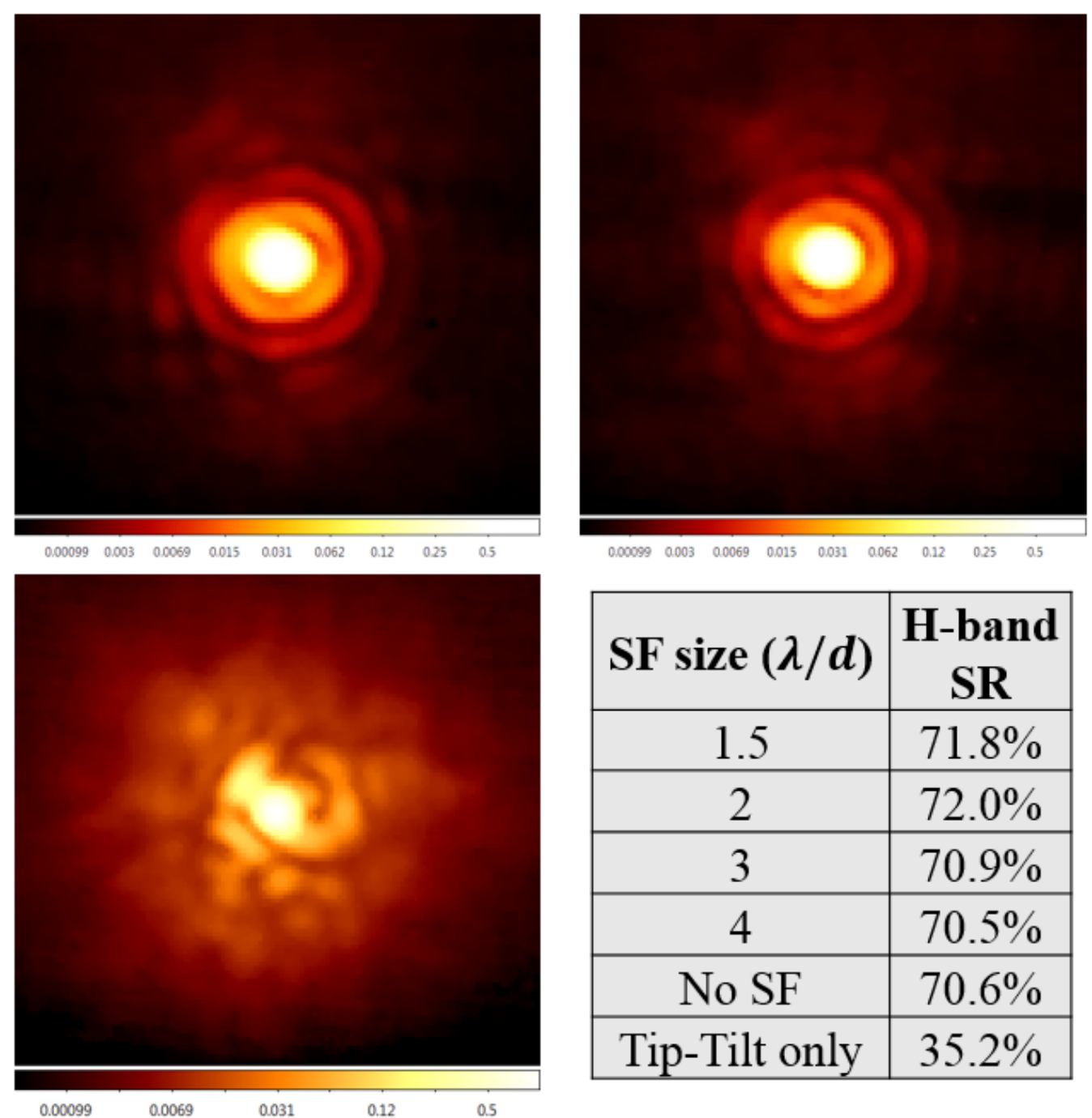

\begin{tabular}{|c|c|}
\hline SF size $(\boldsymbol{\lambda} / \boldsymbol{d})$ & $\begin{array}{c}\text { H-band } \\
\text { SR }\end{array}$ \\
\hline 1.5 & $71.8 \%$ \\
\hline 2 & $72.0 \%$ \\
\hline 3 & $70.9 \%$ \\
\hline 4 & $70.5 \%$ \\
\hline No SF & $70.6 \%$ \\
\hline Tip-Tilt only & $35.2 \%$ \\
\hline
\end{tabular}

Figure 7: Normalized PSFs of different spatial filter setups in H-band. Logarithmic colorbar. Top-left: no spatial filter. Top-right: $1.5 \lambda / d$ spatial filter. Bottom-left: Tip-tilt AO only and no spatial filter (for reference). 


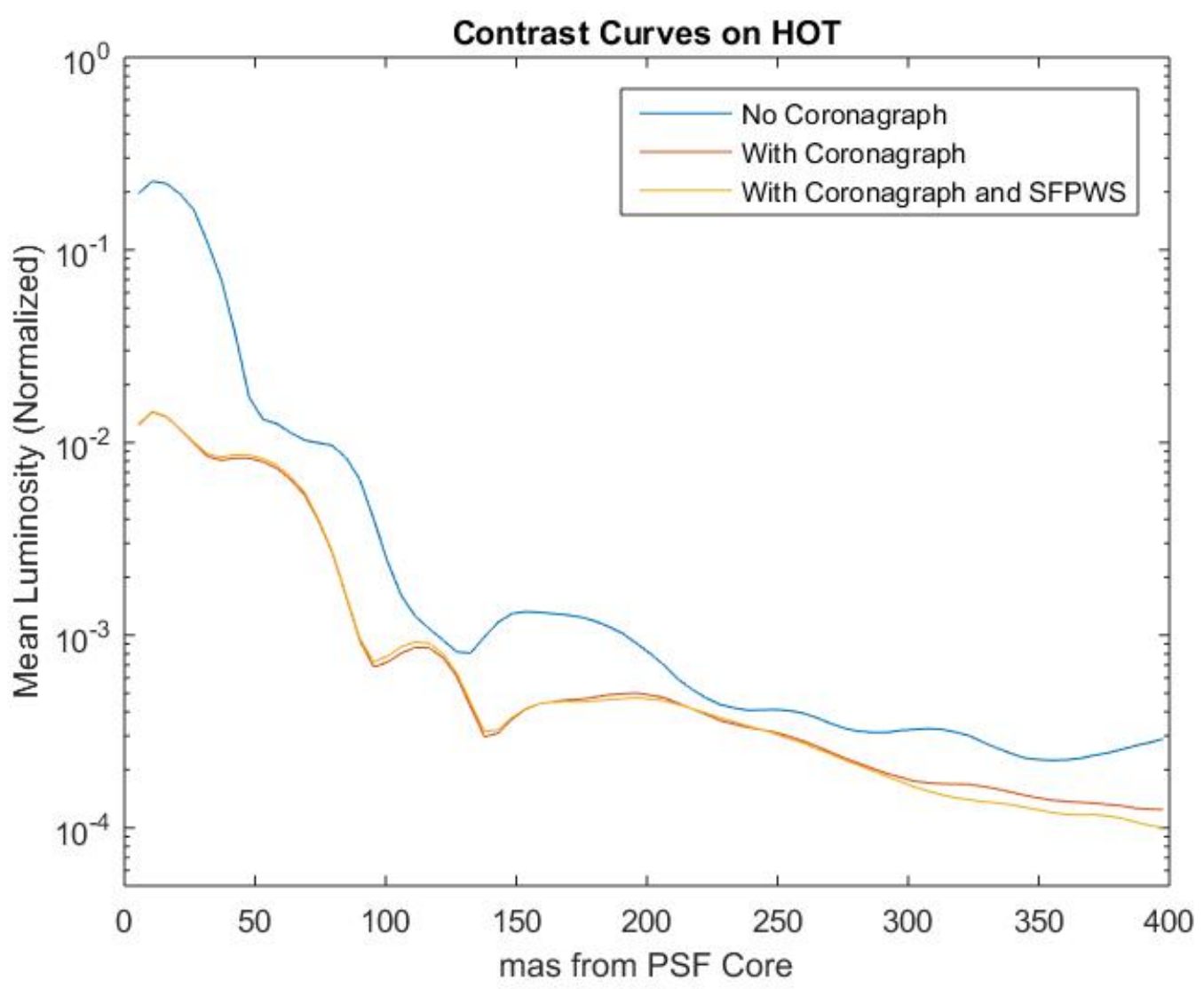

Figure 8: Contrast curves on HOT with and without a coronagraph and spatially filtered PWS. The decrease in peak flux and changed Airy ring structure indicate that the coronagraph is functioning properly. Also, note a slight improvement in the achieved contrast depth in the spatially filtered PWS case.

H-band PSFs were recollected on the HOT testbench using the same conditions outlined in Section 3. The plot in Figure 8 shows the results. There are two observations which indicated that the coronagraph is functioning, although perhaps not as well as one would expect. First of all, the peak flux in the PSF core drops by more than an order of magnitude when the coronagraph is in place; unfortunately, other coronagraphs can supress on-axis light by a factor of several hundred. Second, the Airy ring structure is shifted when the coronagraph is used, which is a characteristic effect of coronagraphs.

The correctable region of the AO system on HOT extends to about 400 mas on HOT. This is the region where one could expect aliasing to have its greatest impact. Indeed, in this region, the spatially filtered PWS (yellow line) shows a slight increase in the achieved contrast depth relative to the not spatially filtered PWS (red line). As with the Strehl ratio analysis, however, this is quite a negligible improvement.

\section{CONCLUSION AND PERSPECTIVES}

Closed loop tests were performed in the presence of turbulence to investigate to which degree PWS are susceptible to aliasing. Performance was evaluated according to two metrics, Strehl ratio and contrast, where the second data set was gathered using a vortex coronagraph. Results show that aliasing is not a dominant factor in the PWS error budget, which agrees with simulations by Vérinaud et al., 2004 and Bond, 2016. ${ }^{2,3}$

\subsection{Sensitivity}

Ragazonni, 2017 has suggested that spatial filtering on a PWS may be able to increase the sensitivity of a PWS (thus helping the wavefront sensor to track fainter guide stars) by only allowing photons that carry use- 
ful information to reach the detector. ${ }^{8}$ This means that no signal at all would be detected by a perfectly flat wavefront. This technique is coined "dark wavefront sensing," and is an extension of the spatial filtering experiment described here. Similarly, wavefront sensing using multiple spatial frequencies provides could also extend PWS performance. HOT provides a highly adequate environment for performing experiments in dark wavefront sensing and multiple spatial frequency sensing using a PWS.

\section{REFERENCES}

[1] Poyneer, L. A. and Macintosh, B., "Spatially filtered wave-front sensor for high-order adaptive optics," $J$. Opt. Soc. Am. 21, 810-819 (2004).

[2] Vérinaud, C., Le Louarn, M., Korkiakoski, V., and Braud, J., "Simulations of extreme AO: a comparison between shack-hartmann and pyramid-based systems," in [Advancements in Adaptive Optic], Proc. SPIE 5490 (2004).

[3] Bond, C., "Real-time pyramid WF reconstruction in the fourier domain: Preparing simulations for elts," in [Wavefront Sensing in the VLT/ELT Era], (2016).

[4] Kasper, M., "Adaptive optics for high-contrast imaging," in [Adaptive Optics Systems III], Proc. SPIE $\mathbf{8 4 4 7}$ (2012).

[5] Saleh, B. E. A. and Teich, M. C., [Fundamentals of photonics], Wiley, New York (1991).

[6] Aller-Carpentier, E., "Extreme Adaptive Optics system optimization for High Contrast Imaging with the High Order Test bench." PhD Thesis at Universidad Autnoma de Madrid (2011).

[7] Poyneer, L. A., Rosa, R. J. D., Macintosh, B., and et al, "On-sky performance during verification and commissioning of the Gemini Planer Imager's adaptive optics system." arXiv https://arxiv.org/pdf/ 1407.2278.pdf (2014).

[8] Ragazonni, R., "Multiple spatial frequencies wavefront sensing," in [AO4ELT5 Conference Proceedings], (2017). 